\title{
The Impact of Oxygen Vacancies on Lithium Vacancy Formation and
}

\author{
Diffusion in $\mathrm{Li}_{2-x} \mathrm{MnO}_{3-\delta}$ \\ Christine James ${ }^{1}$, Yan $\mathrm{Wu}^{2}$, Brian W. Sheldon ${ }^{3}$, Yue Qi ${ }^{1 *}$ \\ ${ }^{1}$ Department of Chemical Engineering and Materials Science, Michigan State University, East \\ Lansing, MI,48824 \\ ${ }^{2}$ General Motors Global Research \& Development Center, 30500 Mound Road, Warren, MI \\ 48090 \\ ${ }^{3}$ School of Engineering, Brown University, Providence, RI 02912
}

\begin{abstract}
High-capacity battery cathode materials containing $\mathrm{Li}_{2} \mathrm{MnO}_{3}$ appear to be activated during the first electrochemical cycle through the generation of oxygen vacancies. These oxygen vacancies have been predicted to alter the atomistic scale structure of $\mathrm{Li}_{2} \mathrm{MnO}_{3}$ and thus impact the delithiation process. In order to understand the effects of these oxygen vacancies we computationally determine the location of lithium removal in $\mathrm{Li}_{2} \mathrm{MnO}_{3-\delta}(\delta=0,0.0625$ and 0.125$)$ and study lithium diffusion in $\mathrm{Li}_{2-\mathrm{x}} \mathrm{MnO}_{3-\delta}$. This study shows that it is energetically favorable for the lithium vacancies to form in the neighboring positions of the oxygen vacancies, suggesting that there is a strong interaction between the two vacancy types. This interaction also inhibits the diffusion of the lithium-ions, as seen in ab-initio molecular dynamics simulations where less hopping of lithium atoms is observed when oxygen vacancies are present. Additionally, the oxygen vacancy containing structures lead to an increase in the energy barrier for lithium hopping and the calculated diffusion coefficient decreases by $\sim 5$ orders of magnitude from the perfect crystal structure.
\end{abstract}

*Email: yueqi@egr.msu.edu 


\section{Introduction}

The layered excess lithium transition metal oxides of $x \mathrm{Li}_{2} \mathrm{MnO}_{3}-(1-x) \mathrm{Li}(\mathrm{Ni}, \mathrm{Co}, \mathrm{Mn}) \mathrm{O}_{2}$ have gained much attention due to their large reversible capacity $(>200 \mathrm{mAh} / \mathrm{g})(1)$ compared to other common cathode materials for lithium-ion batteries (LIBs) (2-5), such as $\mathrm{LiCoO}_{2}(\sim 160 \mathrm{mAh} / \mathrm{g})$, $\mathrm{LiFePO}_{4}(\sim 160 \mathrm{mAh} / \mathrm{g})$ and $\mathrm{LiMn}_{2} \mathrm{O}_{4}(\sim 130 \mathrm{mAh} / \mathrm{g}) .(6)$ This large reversible capacity is generally believed to come from the "activation" process of the $\mathrm{Li}_{2} \mathrm{MnO}_{3}$ component. This "activation" is observed at $>4.4 \mathrm{~V}(1)$, which is necessary to access the $\mathrm{Li}$ in the $\mathrm{Li}_{2} \mathrm{MnO}_{3}$ phase.(7-9) While this material is promising, it suffers from problems such as structural instability, hysteresis, voltage decay (10-12), low rate capability (13), and poor life. These problems have prevented its practical applications.

Although there are still some arguments on whether $x \mathrm{Li}_{2} \mathrm{MnO}_{3}-(1-x) \mathrm{Li}(\mathrm{Ni}, \mathrm{Co}, \mathrm{Mn}) \mathrm{O}_{2}$ is mixed as a solid solution or segregated in separate domains $(1,14)$, it is unarguably important to understand how the $\mathrm{Li}$ in the $\mathrm{Li}_{2} \mathrm{MnO}_{3}$ component contributes to the observed high capacity. The $\mathrm{Li}_{2} \mathrm{MnO}_{3}$ phase was initially thought to be electrochemically inactive due to the high oxidation state $(4+)$ of the $\mathrm{Mn}$ cation, thus inhibiting access to the $\mathrm{Li}$ cations. However, the $\mathrm{Li}_{2} \mathrm{MnO}_{3}$ component alone (15-24) and $\mathrm{Li}_{2} \mathrm{TMO}_{3}$ layered compounds (25), where $\mathrm{TM}$ is a combination of $\mathrm{Mn}$ and other transition metals or other transition metals, have been proven as high capacity cathodes as well. In them, an activation process is also necessary.

Several mechanisms have been suggested for the process of activating the $\mathrm{Li}_{2} \mathrm{MnO}_{3}$ component, such as oxidation of the $\mathrm{O}^{2-}$ to $\mathrm{O}^{-}$ions $(26,27)$, the loss of oxygen $(1,17,23,27)$, the exchange of Li ions for $\mathrm{H}$ ions from the electrolyte (17), and possibly the oxidation of $\mathrm{Mn}^{4+}$ (15). Several studies have modeled the $\mathrm{Li}$ removal process in $\mathrm{Li}_{2} \mathrm{MnO}_{3}$ to mimic the activation process of $\mathrm{Li}_{2}$ ${ }_{x} \mathrm{MnO}_{3} \cdot(28-32)$. These studies detailed the sequence of Li being removed from either Li layer or 
transition metal layer, and proved that it is energetically favorable to release $\mathrm{O}_{2}$ after a large amount of Li being removed (such as $x>1$ (28)). Thus, the oxygen loss contribution to the increase in capacity is still one of the most important and commonly accepted mechanisms, no matter whether the oxygen is lost from the bulk or the surface of the cathode. Lee and Persson (28) suggested that the oxygen diffusion barrier is too large for oxygen to be removed from the bulk and therefore it is removed from the surface. Oxygen vacancies can also be formed during material synthesis, as suggested by experiments (33) and modeling (34). Regardless, it is likely that oxygen vacancies facilitate the high capacity, such that the cathode material should be written as $\mathrm{Li}_{2-x} \mathrm{MnO}_{3-\delta}$ during electrochemical cycling, where $\delta$ denotes the oxygen nonstoichiometry. To improve the subsequent durability and performance of this material after the activation process, we must understand how oxygen vacancies in $\mathrm{Li}_{2} \mathrm{MnO}_{3-\delta}$ impact the delithiation process, structural change, and the rate performance of the $\mathrm{Li}_{2-x} \mathrm{MnO}_{3-\delta}$ phase.

It has been demonstrated that oxygen vacancies can change the transition metal diffusion. It is well established that degradation of the Li-excess layered $x \mathrm{Li}_{2} \mathrm{MnO}_{3}-(1-x) \mathrm{Li}(\mathrm{Ni}, \mathrm{Co}, \mathrm{Mn}) \mathrm{O}_{2}$ phase is related to the transformation from layered to spinel $(14,35-38)$ which starts from Mn diffusion from the transition metal layer to the Li layer (39). Computational studies have shown that the oxygen vacancies decrease the energy barrier for the transition metal migration $(40,41)$. Because the spinel phase has lower energy and capacity than the layered phase, this is likely to be the fundamental reason for the voltage decay and hysteresis observed in this material. While various methods (1)(5) are pursed to prevent the phase transformation and maintain a layered structure, another technical challenge, its low intrinsic rate performance (13), is still unsolved.

It is not clear if oxygen vacancies will alter Li diffusion as well. Practically, $x \mathrm{Li}_{2} \mathrm{MnO}_{3}-(1-$ $x) \mathrm{Li}(\mathrm{Ni}, \mathrm{Co}, \mathrm{Mn}) \mathrm{O}_{2}$ also has the disadvantage of low intrinsic rate performance (13). Without the 
proper kinetics, the high voltage and high capacity region cannot be accessed in practical applications. For example $x \mathrm{Li}_{2} \mathrm{MnO}_{3}-(1-x) \mathrm{Li}(\mathrm{Ni}, \mathrm{Co}, \mathrm{Mn}) \mathrm{O}_{2}$ exhibits very high resistance at low state of charge (SOC) (42), where the resistance increases rapidly and lowers the usable energy obtained with normal charge-discharge conditions in practical applications. This dramatic resistance increase occurs at lower $\mathrm{SOC}$, when the $\mathrm{Li}_{2-\mathrm{x}} \mathrm{MnO}_{3-\delta}$ participates in delithiation/lithiation. Galvanostatic intermittent titration technique with electrochemical impedance spectroscopy measurements (42) and direct observation on the reaction kinetics for each element (13) clearly suggested that the underlying high resistance is caused by the $\mathrm{Li}_{2-}$ ${ }_{x} \mathrm{MnO}_{3-\delta}$ phase after the activation process. With the existing experimental evidences, it is still not possible to determine whether slow electron or slow Li ion transport is responsible for the high resistance. However, the computed band gap for $\mathrm{Li}_{2} \mathrm{MnO}_{3}$ is $\sim 2.1 \mathrm{eV}$ (29) (comparable to $\mathrm{LiCoO}_{2} \sim 2.7 \mathrm{eV}$ ), thus the high resistance is more likely to be caused by slower $\mathrm{Li}$-ion diffusion. Xiao et al. studied $\mathrm{Li}$ diffusion within $\mathrm{Li}_{2} \mathrm{MnO}_{3}$ using ab-initio molecular dynamics (AIMD) simulations, without considering any vacancies and at $3000 \mathrm{~K}$, a temperature appeared to melt the lattice.(29) However, the oxygen and lithium vacancies may be strongly correlated, making a computational study of the kinetics of the $\mathrm{Li}_{2-x} \mathrm{MnO}_{3-\delta}$ component of great interest. Shi et al. have discovered that $\mathrm{Li}$ diffusion is strongly correlated with neighboring oxygen ions in $\mathrm{Li}_{2} \mathrm{CO}_{3}$ $(43,44)$, more specifically, lower oxygen coordination around the moving $\mathrm{Li}$ ions leads to higher diffusion energy barrier. This suggest that losing oxygen in $\mathrm{Li}_{2-x} \mathrm{MnO}_{3-\delta}$ may hinder $\mathrm{Li}$ diffusion and thus it is important to study Li diffusion kinetics with and without oxygen vacancies.

As discussed above, oxygen vacancies (although the actual amount may be unknown) generated during material synthesis and/or the "activation" process can have a profound impact on the durability and performance of $\mathrm{Li}_{2} \mathrm{MnO}_{3-\delta}$. Therefore, it is critical to understand the effect of 
oxygen non-stoichiometry $(\delta)$ on the structural change and diffusion in $\mathrm{Li}_{2-x} \mathrm{MnO}_{3-\delta}$ and related compounds. Although the simultaneous removal of lithium and oxygen as $\mathrm{Li}_{2} \mathrm{O}$ has been widely used to estimate $\delta$, Meng et al. took this estimation further and compared it to a Rietveld refinement of the structure after cycling and suggested that $\delta$ was significantly less than the amount required to give the observed extra capacity, assuming oxygen vacancies were formed as $\mathrm{Li}_{2} \mathrm{O}$. (45). Experimentally, the investigations of oxygen non-stoichiometry in these materials have been limited by the lack of in-situ measurements of the non-stoichiometry as a function of the state of charge (i.e., Li content). Computationally, on the other hand, it is quite easy to control the oxygen non-stoichiometry, $\delta$, and study of the effect of oxygen non-stoichiometry, $\delta$, on Li vacancy formation and $\mathrm{Li}$ diffusion, providing the insight on the capacity and rate performance of this material.

Therefore, this study presented here is focused on using DFT calculations to analyze the formation of oxygen and lithium vacancies individually in section 3.1 and the effects of oxygen vacancies on the delithiation process of $\mathrm{Li}_{2-x} \mathrm{MnO}_{3-\delta}$ in section 3.2. Additionally, this study used AIMD to track $\mathrm{Li}$ diffusion in $\mathrm{Li}_{2-x} \mathrm{MnO}_{3-\delta}$ at $1500 \mathrm{~K}$ to avoid melting of the system and to analyze the effects of oxygen vacancies on Li diffusion in section 3.3. The energy barriers of lithium diffusion was additionally calculated to estimate the diffusion coefficients with and without oxygen vacancies in section 3.3. Section 3.4 contains open circuit voltage (OCV) calculation results and discussions on how $\mathrm{V}_{O}$ changes the subsequent capacity, voltage, and diffusion in $\mathrm{Li}_{2-x} \mathrm{MnO}_{3-\delta}$ and related compounds.

\section{Computational Details}


The Vienna $A b$ initio Simulation Package (VASP) (46-49) was used to perform plane wave density functional theory (DFT) calculations. Potentials constructed with the full potential projector augmented wave (PAW) method $(50,51)$ were used for the elemental constituents. The exchange-correlation part of the density functional was treated with the generalized gradient approximation (GGA) as parameterized by Perdew, Burke and Ernzerhof $(52,53)$ with a Hubbard $\mathrm{U}$ correction (so called GGA+U method). The U parameter was chosen to be $4.84 \mathrm{eV}$ for Mn, a similar value to literature.(29-31) Valence electron configurations for the elemental constituents were as follows: $\mathrm{Li}-1 \mathrm{~s}^{2} 2 \mathrm{~s}^{1}, \mathrm{Mn}-4 \mathrm{~s}^{2} 3 \mathrm{~d}^{5}$, and $\mathrm{O}-2 \mathrm{~s}^{2} 2 \mathrm{p}^{4}$. In all calculations, the electronic degrees of freedom were converged to $10^{-6} \mathrm{eV}$. During ionic position optimization, the Hellmann-Feynman force components on each atom were relaxed to $0.02 \mathrm{eV} \AA^{-1}$.

Prior to the vacancy calculations, the structure (lattice parameters and ionic positions) of the conventional cell of $\mathrm{Li}_{2} \mathrm{MnO}_{3}(\mathrm{C} 2 / \mathrm{m})$ were optimized, as shown in Figure 1. For this purpose, a plane-wave basis cutoff energy of $550 \mathrm{eV}$ and a k-points mesh of $3 \times 3 \times 3$ for Brillouin zone sampling were found to be adequate to give an energy convergence of $0.2 \mathrm{meV} / \mathrm{atom}$ for the primitive cell. The minimized lattice parameters are listed in Table 1 and compared with literature values determined by calculation and experiments. Our calculated lattice parameters are only less than $2 \%$ different from the experimental values, likely to be due to the under binding of GGA, validating the parameters used in DFT calculations.

All reported vacancy calculations were performed on the $2 \times 1 \times 2$ supercells (96 atoms for perfect $\mathrm{Li}_{2} \mathrm{MnO}_{3}$ ). From this starting structure $\mathrm{Li}_{2} \mathrm{MnO}_{3}$, one or two oxygen atoms were removed to form oxygen vacancy (Vo) containing structures as $\delta=0.0625$ and 0.125 in $\mathrm{Li}_{2} \mathrm{MnO}_{3-\delta}$, respectively. A continuous lithium vacancy $\left(\mathrm{V}_{\mathrm{Li}}\right)$ generation simulation was designed to mimic the delithiation process at different oxygen vacancy concentrations. To be more general, we note 
the simulation structure as $\mathrm{Li}_{2-x} \mathrm{MnO}_{3-\delta}$, where the lithium vacancy concentration $x$ increases, as $x$ $=0,0.0625,0.1250,0.1875$ and 0.2500 , while the oxygen vacancy concentration $\delta$ varies, as $\delta=$ $0,0.0625$ and 0.1250 . At a given $\delta$ value, the continuous delithiation simulation was performed by generating various new configurations with one more Li vacancy added to the minimized structure for the previous $x$ value, until a configuration with minimized energy is found. This process repeats itself to increase $x$ values. Although, there are many possible configurations at a given $x$ and $\delta$ combination, a general trend between Li vacancy formation energy and its distance from the oxygen vacancy was discovered. This relationship allows us to identify quickly the configurations with the lowest energy, instead of sampling all possible vacancy arrangements. In fact, calculations on a smaller $2 \times 1 \times 1$ supercell allowed sampling all possible lithium positions at each concentration, suggesting the same trend. More specific details of the sampling and the minimized configurations are discussed in sections 3.1 and 3.2. The configurations with the lowest energies at each $x$ and $\delta$ in $\mathrm{Li}_{2-x} \mathrm{MnO}_{3-\delta}$ was taken as the minimized structure for further analysis and the energies were used to compute the formation energy. Assuming one more Li is removed from $E_{L_{2-x_{1}} \mathrm{MnO}_{3-\delta}}$ to form $E_{L_{2-x_{2}} \mathrm{MnO}_{3-\delta}}$, or in other words, the Li extraction from the structure with $x_{1}$ amount of lithium initially leads to the final structure with $x_{2}$ amount of lithium removed, the $\mathrm{V}_{\mathrm{Li}}$ formation energy was calculated as

$$
E_{f, \text { Livacancy }}=E_{L_{2-x_{2}} M n O_{3-\delta}}+E_{L i, \text { metal }}-E_{L i_{2-x_{1}} M n O_{3-\delta}}
$$

To investigate the impact of oxygen vacancy on the diffusion of Li, ab inito molecular dynamics (AIMD) implemented in VASP were used. The AIMD simulations were performed on $2 \times 1 \times 2$ cells of approximately 96 atoms at $1500 \mathrm{~K}$ using the Nosé (54) thermostat. Systems of $\mathrm{Li}_{2-}$ ${ }_{x} \mathrm{MnO}_{3-\delta}$ were analyzed for $x=0.0625,0.1250$ and $\delta=0,0.0625$. Diffusion was studied by tracking the displacement of the lithium and oxygen atoms from their initial positions. 
Additionally, the climbing image nudged elastic band (cNEB) method implemented in VASP was used to calculate energy barriers for lithium diffusion. $(55,56)$

\section{Results and Discussion}

\subsection{Separate Oxygen Vacancy and Lithium vacancy formation}

Before investigating the impact of oxygen vacancies on the formation of lithium vacancies, the location of the oxygen vacancies must be determined. The first $\mathrm{V}_{\mathrm{O}}$ was determined by calculating the energy of two systems with either a $\mathrm{V}_{\mathrm{O}}$ at the $8 j$ or $4 i$ position (unique Wyckoff positions for $\mathrm{O}$ ) and comparing the system energies. It was found that the first $\mathrm{V}_{\mathrm{O}}$ on the $8 j$ site was more energetically favorable by $0.36 \mathrm{eV}$. However, this was unexpected at a first glance, since the bond length between the $8 j$ oxygen ion and Mn was shorter than the bond length between the $4 i$ oxygen ion and $\mathrm{Mn}$. Therefore, we estimated the electrostatic energy ( $E_{\text {electrostatic }}$ ) of both positions of oxygen using their nearest neighboring four $\mathrm{Li}$ and two $\mathrm{Mn}$ ions, their respective charges $\left(q_{L i}, q_{M n}, q_{O}\right)$ and the distance between the oxygen and the neighboring atom $(r)$.

$$
E_{\text {electrostatic }}=q_{O}\left(\sum \frac{q_{L i}}{r}+\sum \frac{q_{M n}}{r}\right)
$$

Thus, the oxygen ion at the $4 i$ position had a more attractive electrostatic energy than the oxygen ion at the $8 j$ position by $0.05 \mathrm{eV}$ or $0.17 \mathrm{eV}$, when the classical or Bader point charges was used, respectively. Thus, it will be more energetically favorable to remove the oxygen at the $8 j$ position. This observation is consistent with previous calculations done by Okamoto et al., showing that $\mathrm{V}_{\mathrm{O}}$ at the $8 j$ position is energetically more favorable than the $4 i$ position by $0.4 \mathrm{eV}$. The predicted $\mathrm{V}_{\mathrm{O}}$ formation energy (on $8 j$ site) is $2.1 \mathrm{eV}$. For the system with two $\mathrm{V}_{\mathrm{O}}$, or $\delta=0.1250$, the most energetically favorable position for the second $\mathrm{V}_{\mathrm{O}}$ was in an $8 j$ position $3.1 \AA$ 
from the first $V_{0}$. The energy of formation of the second vacancy was $0.05 \mathrm{eV}$ smaller than the energy of formation of the first oxygen vacancy, and thus $0.05 \mathrm{eV}$ smaller than the energy required to remove a second oxygen atom far from the initial $V_{O}$.

From the system with no $\mathrm{V}_{\mathrm{O}}$, a lithium atom from each unique Wyckoff position $(4 h, 2 b$, and $2 c$ shown in Figure 1) was removed. A DFT calculation was performed to relax the atomic positions and the system energies were compared. For the system without $V_{O}$ the $V_{L i}$ were formed in the 4h position, consistent with Okamoto.(31) The $\mathrm{V}_{\mathrm{Li}}$ at the $4 h$ position was more favorable than the $\mathrm{V}_{\mathrm{Li}}$ at the $2 b$ position by $0.18 \mathrm{eV}$ and $0.05 \mathrm{eV}$ more favorable than the $\mathrm{V}_{\mathrm{Li}}$ at the $2 \mathrm{c}$ position. Due to the small energy difference between these positions, it is likely that Li can sample all of these positions at room temperature.

\subsection{Impact of Oxygen Vacancies on Lithium Vacancy Formation}

For the systems with oxygen vacancies, lithium vacancies were introduced into the system one at a time. Preliminary simulations on a $2 \times 1 \times 1$ supercell suggested that lithium vacancies tend to form near oxygen vacancies. Therefore, the sampled structures include lithium atoms being removed from all possible positions near the $\mathrm{V}_{\mathrm{O}}$ and a few more positions farther away to confirm the trend. The formation energy of the last generated $\mathrm{V}_{\mathrm{Li}}$ according to Equation (1) are correlated with the distance between $V_{O}$ and $V_{L i}$ in Figure 2. For the system with one $V_{O}$ the distance was defined using the optimized coordinates of the oxygen site in the perfect cell and the optimized coordinates of the $\mathrm{Li}$ atom in the oxygen vacancy containing structure prior to it being removed. When there are two oxygen vacancies, the center position of the two oxygen atoms in the perfect cell was used to compute the distance with the Li vacancy. 
From Figure 2a it is apparent that the formation energy for $\mathrm{V}_{\mathrm{Li}}$ in the first nearest neighbor sites is the lowest and the formation energy for the $V_{\mathrm{Li}}$ far away from the $\mathrm{V}_{\mathrm{O}}$ appears to plateau at an energy which is much higher. However, when the third and fourth $\mathrm{V}_{\mathrm{Li}}$ are formed, the formation energy difference between the $V_{\mathrm{Li}}$ far away or near the $\mathrm{V}_{\mathrm{O}}$ diminishes, suggesting that the effect of the $\mathrm{V}_{\mathrm{O}}$ becomes more delocalized as more lithium is removed. Additionally, the $\mathrm{V}_{\mathrm{Li}}$ which formed in the second nearest neighbor positions, seen in Figure $2 \mathrm{~b}$ at around $3.5 \AA$ away from the $V_{\mathrm{O}}$, have an energy higher than the energy at the plateau. This is thought to be because as the first $\mathrm{Li}$ nearest neighbors to the $\mathrm{V}_{\mathrm{O}}$ repel each other and move farther away from the $\mathrm{V}_{\mathrm{O}}$, while the second nearest Li neighbors move slightly towards the $\mathrm{V}_{\mathrm{O}}$ and towards the oxygen they share with the first nearest neighbor Li positions. This structural relaxation makes the second nearest Li neighbors to $\mathrm{V}_{\mathrm{O}}$ more strongly bonded to oxygen, thus energetically less favorable to remove.

A similar analysis was performed for the starting system with two $V_{O}$ from which lithium atoms were removed in Figure $2 \mathrm{~b}$. This analysis suggest that $\mathrm{V}_{\mathrm{Li}}$ tended to form near the existing two $\mathrm{V}_{\mathrm{O}}$ initially as the energetically favored $\mathrm{V}_{\mathrm{Li}}$ formation energies mostly increased with distance between the $\mathrm{V}_{\mathrm{O}}$ and $\mathrm{V}_{\mathrm{Li}}$ and plateaued at large distances. This trend was consistent with the results on a smaller supercell. However, as the third and fourth $V_{\mathrm{Li}}$ are formed, the dependence of $\mathrm{V}_{\mathrm{Li}}$ on its distance to the $\mathrm{V}_{\mathrm{O}} \mathrm{S}$ is diminishing, as the formation energy of $\mathrm{V}_{\mathrm{Li}}$ near $\mathrm{V}_{\mathrm{O}}$ is significantly closer to the formation energy of $\mathrm{V}_{\mathrm{Li}}$ far away from $\mathrm{V}_{\mathrm{O}}$. The formation energy of $\mathrm{V}_{\mathrm{Li}}$ are lower than those in Figure 2a with only one $V_{\mathrm{O}}$, suggesting the two $\mathrm{V}_{\mathrm{O}}$ have a synergistic effect on the $\mathrm{V}_{\mathrm{Li}}$ formation.

The configurations with the lowest Li vacancy formation energies were plotted in Figure 3 with the $\mathrm{V}_{\mathrm{Li}}$ colored yellow and labeled with the numbers indicating the order in which they are removed. It appeared that for the starting systems with $V_{O}$, the $V_{L i}$ tended to cluster near the 
present $\mathrm{V}_{\mathrm{O}}$. When there is only one oxygen vacancy, the first $\mathrm{Li}$ to be removed comes from the Li layer (site $4 h$ ), and the second comes from the transition metal layer (site $2 b$ ), and the third and fourth come from site $2 c$ and $4 h$ in the Li layer (Figure $3 \mathrm{a}$ ). The two $\mathrm{V}_{\mathrm{Li}}$ form a dumbbell structure around the oxygen vacancy, noted as $\mathrm{V}_{\mathrm{Li}}-\mathrm{V}_{\mathrm{O}}-\mathrm{V}_{\mathrm{Li}}$. When there are two oxygen vacancies, the first two Li atoms were removed from the Li metal layer and then transition metal layers, while the final configuration shows two $\mathrm{V}_{\mathrm{Li}}-\mathrm{V}_{\mathrm{O}}-\mathrm{V}_{\mathrm{Li}}$ dumbbell structures. These lithium sites closest to the $\mathrm{V}_{\mathrm{O}}$ are the easiest to be removed during delithiation, but the last to be filled during lithiation due to the formation energy difference.

\subsection{Impact of Oxygen Vacancies on Lithium Diffusion}

AIMD simulations were performed on a few energetically favorable configurations of $\mathrm{Li}_{2}$ ${ }_{x} \mathrm{MnO}_{3-\delta}$, as determined by DFT calculations. These initial configures are listed in Figure 4 and they either contained a single $\mathrm{V}_{\mathrm{Li}}(x=0.0625$ and $\delta=0)$, a single $\mathrm{V}_{\mathrm{Li}}$ and a single $\mathrm{V}_{\mathrm{O}}(x=0.0625$ and $\delta=0.0625)$, or two $\mathrm{V}_{\mathrm{Li}}$ and a single $\mathrm{V}_{\mathrm{O}}(x=0.125$ and $\delta=0.0625)$. AIMD was performed at $1500 \mathrm{~K}$. Some literature have reported $\mathrm{Li}$ diffusion simulations at $3000 \mathrm{~K}$ without any $\mathrm{V}_{\mathrm{Li}}(29)$ nor $\mathrm{V}_{\mathrm{O}}$.. However, we noticed that the lattice became disordered or melted at 3000K in AIMD. At $1500 \mathrm{~K}$, most of the atoms are still vibrating around their lattice positons and hopping of atoms can be clearly tracked by analyzing the trajectory (the frequency of atomic structures writing is $0.02 \mathrm{ps}$ ) during the $50 \mathrm{ps}$ of the AIMD simulations.

The system with one $\mathrm{V}_{\mathrm{Li}}$ and no $\mathrm{V}_{\mathrm{O}}$ showed significant displacement of the lithium atoms. The displacement of every lithium atom from its initial positions was tracked and only those which moved significantly are shown in, Figure 5a, while the displacement of others are almost zero indicating vibration and are not shown. Hopping of Li atoms at a step of $\sim 3 \AA$ can be seen in Figure 5a. The trajectory also shows that the $\mathrm{V}_{\mathrm{Li}}$ only moved on the Li layer. Each hopping 
corresponds to one $\mathrm{Li}$ atom switching position with the $\mathrm{Li}$ vacancy. The $\mathrm{V}_{\mathrm{Li}}$, position was determined by comparing the snapshots of the AIMD trajectory to the perfect crystal structure and defining the $\mathrm{V}_{\mathrm{Li}}$ position as the position of the $\mathrm{Li}$ atom missing from the perfect crystal structure. To quantify the movement of $\mathrm{V}_{\mathrm{Li}}$, its position was tracked every five picoseconds and is shown in Figure 5b. One Li hopped away then back (the blue curve in Figure 5a). Other than that, the hopping events of Li and vacancy are well correlated. Multiple vacancy hopping events occurred during 50ps.

Figure $4 \mathrm{~b}$ shows the minimum energy configuration for $\mathrm{V}_{\mathrm{O}}$ and one $\mathrm{V}_{\mathrm{Li}}$ was the configuration where they were in neighboring $8 j$ and $4 h$ Wyckoff positions, respectively. The AIMD results showed little movement of the $V_{\mathrm{Li}}$ in Figure 6a, and mainly vibrational movement of the $\mathrm{V}_{\mathrm{Li}}$, suggesting that it is unfavorable for $\mathrm{Li}$ atoms to be near the $\mathrm{V}_{\mathrm{O}}$ and thus the $\mathrm{V}_{\mathrm{Li}}$ and $\mathrm{V}_{\mathrm{O}}$ vacancies cluster together. On the other hand, it also proved that this configuration is indeed an energetically favorable position for $\mathrm{V}_{\mathrm{Li}}$, and escaping from this position is energetically unfavorable. As $\mathrm{V}_{\mathrm{Li}}$ and $\mathrm{V}_{\mathrm{O}}$ tended to cluster in the most energetically favorable configurations, determined by DFT calculations, these vacancies tended to cluster together even at $1500 \mathrm{~K}$. This led to the $\mathrm{V}_{O}$ blocking diffusion of the lithium atoms. To further demonstrate this effect, a system was setup with the $\mathrm{V}_{O}$ and $\mathrm{V}_{\mathrm{Li}}$ farther apart (Figure 4c). The displacement of the lithium atoms, Figure 6b, and oxygen atoms, Figure $6 \mathrm{c}$, were then tracked. The lithium and oxygen atoms both moved such that the final positions of the lithium and oxygen vacancies were in neighboring layers, back to a clustered $\mathrm{V}_{\mathrm{O}}-\mathrm{V}_{\mathrm{Li}}$ configuration.

The DFT optimized system with the $\mathrm{V}_{\mathrm{Li}}-\mathrm{V}_{\mathrm{O}}-\mathrm{V}_{\mathrm{Li}}$ dumbbell structure (Figure $4 \mathrm{~d}$ ) was also investigated with AIMD. Initially, the $\mathrm{V}_{\mathrm{O}}$ was located at the $8 j$ position and the $\mathrm{V}_{\mathrm{Li}}$ were at a $4 h$ position in the lithium layer, and at a $2 b$ position in the transition metal layer. No significant 
movement, other than vibrations, of the $\mathrm{O}$ atoms was seen. However, there was some significant movement of Li atoms, shown in Figure 6d. The majority of the Li movement was the $2 b \mathrm{~V}_{\mathrm{Li}}$ in the transition metal layer moving to a lithium layer and then hopping within that layer. The only movement of the $4 h \mathrm{~V}_{\mathrm{Li}}$ that neighbored the $8 j \mathrm{~V}_{\mathrm{O}}$ was seen by one Li atom, which moved into that $\mathrm{V}_{\mathrm{Li}}$ for a short time period, seen by the blue line in Figure $6 \mathrm{~d}$. This is an interesting observation, as it may suggest that one $\mathrm{V}_{\mathrm{Li}}$ is much more tightly bounded to the $\mathrm{Vo}$ than the second one, and likely the rest of $\mathrm{V}_{\mathrm{Li}}$, since they are further away from the Vo. This is also consistent with the $\mathrm{V}_{\mathrm{Li}}$ formation energy results, as the formation energy of $\mathrm{V}_{\mathrm{Li}}$ increase with the $x$ and the distance from the Vo. These AIMD results show that fewer or no hopping occurred after oxygen vacancy formation, suggesting oxygen vacancies hinder the diffusion of $\mathrm{Li}$ atoms.

Although multiple hopping events can be observed at $1500 \mathrm{~K}$ with $50 \mathrm{ps}$ AIMD, this timescale is still too short to compute the diffusion coefficient with the mean displacement method. Therefore, we performed cNEB calculations to quantitatively compare the Li vacancy hopping barrier with and without oxygen vacancies. According to the sequences of Li to be removed from the structure with one Vo (Figure 3a), two diffusion paths were computed: Li and vacancy hopping between Wyckoff positions $(4 h, 2 c)$, within the Li layer, and $(2 c, 2 b)$, between the Li layer and the transition metal layer. These cNEB calculations were performed with and without a single $\mathrm{V}_{\mathrm{O}}$, Figure 7 . The energy barrier for $\mathrm{V}_{\mathrm{Li}}$ hopping between the $4 h$ and $2 c$ positions within the lithium layer was higher than the barrier along the hopping pathway between the $2 c$ and $2 b$ positions through the transition metal layer to the lithium layer. This observation is consistent with Xiao's DFT+U results.(29) Additionally, the energy barriers increased significantly due to the nearby $\mathrm{V}_{\mathrm{O}}$. For $\mathrm{V}_{\mathrm{Li}}$ hopping between the $4 h$ and $2 c$ positions, the forward energy barrier increased from $0.78 \mathrm{eV}$ to $1.01 \mathrm{eV}$ and the reverse hopping energy barrier increased from 0.83 
$\mathrm{eV}$ to $1.20 \mathrm{eV}$. For $\mathrm{V}_{\mathrm{Li}}$ hopping between the $2 c$ and $2 b$ positions, the forward energy barrier increased from $0.55 \mathrm{eV}$ to $0.91 \mathrm{eV}$ and the reverse hopping energy barrier increased from 0.69 $\mathrm{eV}$ to $0.98 \mathrm{eV}$. To make comparisons with experimental measurements, the computed hopping barrier, $E_{A}$, and hopping distance, $a$, can be used to calculate diffusion coefficient with a standard random diffusion coefficient via,

$$
D_{r w}=v a^{2} \exp \left(-\frac{E_{A}}{k_{B} T}\right)
$$

where $k_{B}$ is the Boltzmann constant, $T$ is the temperature, and $v$ is the vibrational frequency.

The value of $v$ in Eq. (3) was estimated as $10^{13} \mathrm{~Hz}$, as commonly used in the literature. (57-62) As a comparison to the MD results, the number of hops expected in the 50 ps simulated time period was estimated by using the hopping frequency, $p=v \exp \left(-E_{A} /\left(k_{B} T\right)\right)$. The MD results for the system with one Li vacancy and no oxygen vacancy $(x=0.0625$ and $\delta=0)$ was used as a comparison for simplicity. Based on the $E_{A}$ of Li hopping within the Li layer, because the AIMD simulation showed lithium diffusion on the lithium layer only, it was calculated that $\sim 1$ lithium atom should hop within $50 \mathrm{ps}$ at the simulated $1500 \mathrm{~K}$. This is consistent with the 4 lithium atoms hops observed in the AIMD simulation. This also supports the choice of vibrational frequency $v=10^{13} \mathrm{~Hz}$ for diffusion coefficient calculations. It is also estimated that it would require about $1100 \mathrm{ps}$ for a hop to be observed at $1000 \mathrm{~K}$ and would be computationally too expensive for AIMD. Thus $1500 \mathrm{~K}$ was an appropriate temperature in terms of being high enough to see diffusion in a reasonable computation time and low enough to prevent the structure from melting or being significantly distorted.

$D_{r w}$ was then calculated for both Li ion migrations ( $4 h \leftrightarrow 2 c$ and $2 c \leftrightarrow 2 b$ ) according to Eq. (3). The value of $a$ was estimated to be $2.9 \AA$. The average value of $E_{A}$ was chosen as an average of 
the forward and reverse hopping barrier between the $4 h$ and $2 c$ positions, about $0.81 \mathrm{eV}$ with no $\mathrm{V}_{\mathrm{O}}$ and about $1.1 \mathrm{eV}$ for the system with one $\mathrm{V}_{\mathrm{O}}$. At $T=300 \mathrm{~K}$, the calculated $D_{r w}$ is $2.5 \times 10^{-16}$ $\mathrm{cm}^{2} \mathrm{~s}^{-1}$ for the system with no $\mathrm{V}_{\mathrm{O}}$ and $2.2 \times 10^{-21} \mathrm{~cm}^{2} \mathrm{~s}^{-1}$ with $\mathrm{V}_{\mathrm{O}}$, five orders of magnitude smaller. For the $2 c \leftrightarrow 2 b$ hopping, the average $E_{A}$ values were again used, about $0.62 \mathrm{eV}$ for the system without $\mathrm{V}_{\mathrm{O}}$ and about 0.95 for the system with one $\mathrm{V}_{\mathrm{O}}$, and the value of $a$ was estimated as 2.9 $\AA$. The value of $D_{r w}$ at $T=300 \mathrm{~K}$ was calculated to be $3.3 \times 10^{-13} \mathrm{~cm}^{2} \mathrm{~s}^{-1}$ without $\mathrm{V}_{\mathrm{O}}$ and $1.01 \times 10^{-18} \mathrm{~cm}^{2} \mathrm{~s}^{-1}$ with $\mathrm{V}_{\mathrm{O}}$ present, five orders of magnitude lower. Note we only used the diffusion barrier near the $\mathrm{V}_{\mathrm{O}}$ to estimate the $D_{r w}$ for the system with $\mathrm{V}_{\mathrm{o}}$, if the $\mathrm{V}_{\mathrm{Li}}$ hopping occurs far away from the $V_{O}$, the effect of Vo will be reduced. Thus, this estimation represents the lower bound of $D_{r w}$ in a $\mathrm{V}_{\mathrm{O}}$ containing structure. The estimated $D_{r w}$ for the system without oxygen vacancies is smaller compared to the estimated value of $4.78 \times 10^{-11} \mathrm{~cm}^{2} \mathrm{~s}^{-1}$ determined for uncycled $\mathrm{Li}_{2} \mathrm{MnO}_{3}$ from muon-spin rotation and relaxation experiments $(63,64)$ but closer to $10^{-}$ ${ }^{14} \sim 10^{-18} \mathrm{~cm}^{2} \mathrm{~s}^{-1}$ obtained for $0.5 \mathrm{Li}_{2} \mathrm{MnO}_{3}-0.5 \mathrm{LiMn}_{0.42} \mathrm{Ni}_{0.42} \mathrm{Co}_{0.16} \mathrm{O}_{2}$ at higher activation voltage (in the activated $\mathrm{Li}_{2} \mathrm{MnO}_{3}$ phase). (42)

Using $D_{r w}$ to interpret the computational results corresponds to a lower bound value, since it assumes oxygen vacancies impact all of the atomic jumps. While this permitted the approximate comparisons above, it is important to note that simple random walk statistics are not valid here (i.e. if the oxygen vacancies have the predicted effect on diffusion). Specifically, the oxygen vacancies should lead to correlation effects. In general, $D=f D_{r w}$, where the correlation factor, $f$, describes deviations from random walk behavior (65). Even a standard substitutional diffusion of a tracer atom based on random vacancy motion leads to $f=0.56$ for the two dimensional hexagonal lattice considered below (65): , because of correlations between the motion of the tracer and a neighboring vacancy. When $f$ is this large it is often neglected, however, in $\mathrm{Li}_{2-}$ 
${ }_{x} \mathrm{MnO}_{3-\delta}$, the predicted changes in both the formation energy and the mobility of $\mathrm{Li}$ vacancies will potentially lead to much larger correlation effects. A full analysis of these effects in different crystallographic directions is beyond the scope of this paper. However, to demonstrate the implications of oxygen vacancies on Li diffusion, it is instructive to consider Li diffusion in the Li layers as a relatively simple example (i.e. a $2 \mathrm{D}$ problem). A simplified treatment can be obtained by defining only sites that are fully surrounded by occupied oxygen positions (A sites) and those that are adjacent to an oxygen vacancy (B sites). The Li diffusion coefficient in this plane can then be described as (65):

$$
\begin{aligned}
& D=\frac{\overline{R_{n}^{2}}}{6 t}=\frac{a^{2}}{6} \frac{n}{t} \\
& \frac{n}{t} \cong 6 \omega_{A A}\left[V_{L i}^{\prime}\right]\left(1-\eta_{O}\right) f_{C}\left[\left(\frac{\left(1-\eta_{O}\right)}{1+\frac{\omega_{A B}}{\omega_{A A}} \frac{K_{V O} \eta_{O}}{\left(1-\eta_{O}\right)}}+\frac{K_{V O} \eta_{O}}{\frac{\omega_{B A}}{\omega_{A A}}+\frac{\omega_{B B}}{\omega_{A A}} \frac{K_{V O} \eta_{O}}{\left(1-\eta_{O}\right)}}\right)\right]^{-1} \\
& K_{V O}=\exp \left[\frac{-\left(\Delta G_{V O}^{o}-\Delta G_{V L}^{o}\right)}{k_{B} T}\right]
\end{aligned}
$$

where $n$ is the total number of atomic jumps taken in time, $t,\left[V_{L i}^{\prime}\right]$ is the mole fraction of $\mathrm{Li}$ vacancies, and $\eta_{O}$ is the fraction of the Li sites that are adjacent to oxygen vacancies. In Eq. (5), the frequencies $\omega_{i j}$ refer to jumps between a $\mathrm{Li}$ atom in site $i$ (A or $\mathrm{B}$ ), to a vacant site $j$ (A or B). The thermodynamic quantity $K_{V O}$, is based on the free energies of forming Li vacancies around fully occupied oxygen sites $\left(\Delta G_{V L}^{o}\right)$ and adjacent to a vacant oxygen site $\left(\Delta G_{V O}^{o}\right)$. A factor $f_{C}$ is retained here to describe additional correlation effects that extend beyond the assumptions that lead to Eq. (5). 
Two limiting cases of Eq. (5) are worth noting. In fully stoichiometric material, the limiting diffusion coefficient at $\eta_{O}=0$ is $D(0)=a^{2} \omega_{A A}\left[V_{L i}^{\prime}\right] f_{C}$. With higher $\eta_{O}$, large $\omega_{A A}$ (compared to the other $\omega_{i j}$ ), and the assumption that neighboring oxygen vacancies are not likely (i.e. such that the $\omega_{B B}$ term is negligible), Eqs. (4) and (5) can be used to obtain the following approximation for $f$ :

$$
f\left(\eta_{O}\right)=\frac{D\left(\eta_{O}\right)}{D(0)} \cong \frac{\left(1-\eta_{O}\right)}{K_{V O} \eta_{O}} \frac{\omega_{B A}}{\omega_{A A}}
$$

This result provides a relatively simple demonstration of the expected impact of oxygen nonstoichiometry, $\delta$, on the Li diffusivity. Note here that $\omega_{B A} / \omega_{A A}$ is the same ratio obtained by comparing $D_{r w}$ values with and without oxygen vacancies, where the computational results predicted values of $10^{-5}$ or smaller. The remaining part of the right hand side of Eq. (7) is the ratio of Li vacancies on A and B sites. The DFT results in section 3.2 indicate that $K_{V O}>1$. Thus with $\eta_{O}$ values of several percent, the ratio $\left(1-\eta_{O}\right) / K_{V O} \eta_{O}$ is expected to be on the order 10. While this increases the value of $f\left(\eta_{O}\right)$, the approximation in Eq. (7) still indicates that oxygen vacancies are expected to produce large decreases in the Li diffusivity. A more detailed kinetic Monte Carlo simulation will be used to further explore the Li diffusion as a function of $x$ and $\delta$ value in $\mathrm{Li}_{2-x} \mathrm{MnO}_{3-\delta}$ in the near future.

\subsection{Impact of Oxygen Vacancies on Voltage Curve and resistance}

After understanding how Li was removed with respect to the oxygen vacancy concentration and locations and how oxygen vacancy impact Li diffusion, the DFT determined $\mathrm{V}_{\mathrm{Li}}$ formation energies in the continuous Li removal simulation were used to calculate the OCV. For each $x$ and $\delta$ value in $\mathrm{Li}_{2-x} \mathrm{MnO}_{3-\delta}$ a corresponding OCV was calculated using Equation 8 . The $\mathrm{V}_{\mathrm{Li}}$ formation energies were the lowest energy of formation calculated for each $x$ and $\delta$ combination. 


$$
O C V=\frac{E_{f, \text { Livacancy }}}{e}
$$

These OCV values were then plotted against the calculated theoretical capacity and $x$ values in Figure 8. This figure shows that an increasing amount of $\mathrm{V}_{\mathrm{O}}$ allows for a higher capacity at the same cutoff voltages $(\sim 4.4 \mathrm{~V}$ for example as a practical cutoff voltage to avoid oxidation of the electrolyte). The role of $\mathrm{Li}_{2} \mathrm{MnO}_{3}$ in cycling of excess lithium material $x \mathrm{Li}_{2} \mathrm{MnO}_{3}-(1-$ $x) \mathrm{Li}(\mathrm{Ni}, \mathrm{Co}, \mathrm{Mn}) \mathrm{O}_{2}$ can be understood as the following. During the activation process, $\mathrm{Li}_{2} \mathrm{MnO}_{3}$ loses $x \mathrm{Li}$ and $\delta \mathrm{O}$, becoming $\mathrm{Li}_{2-x} \mathrm{MnO}_{3-\delta}$. Considering $\mathrm{V}_{\mathrm{O}}$ makes neighboring lithium atomic positions energetically less favorable, the $\mathrm{Li}$ atoms near $\mathrm{V}_{\mathrm{O}}$ are the first to be removed. Comparing with the atomic configurations in Figure 3 , it seems the $\mathrm{V}_{\mathrm{Li}}-\mathrm{V}_{\mathrm{O}}-\mathrm{V}_{\mathrm{Li}}$ dumbbell structures is favored, so this may be the structure after $\mathrm{Li}_{2} \mathrm{O}$ release. However, as shown in the OCV curve, the values and the ratio of $x$ and $\delta$ will be determined by the activation voltage. During re-lithiation process, if we consider the oxygen vacancy concentration will not change (although several experimental literature results suggest the change of $\delta$ ), $x$ amount of Li can be re-intercalated. Based on the formation energy, the sites next to $V_{O}$ are the last to be filled. However, if the cutoff voltage is not low enough, less than $x$ amount of Li will be re-intercalated, leaving some Li sites near the $\mathrm{V}_{\mathrm{O}}$ empty. However due to the large hopping barrier of the Li next to the oxygen vacancy, the diffusion becomes sluggish at lower voltage (or lower state of charge) in the following cycles. Considering the AIMD results showing that one $\mathrm{V}_{\mathrm{Li}}$ is much more tightly bounded to the Vo than the rest (Figure $6 \mathrm{~d}$ on two $\mathrm{V}_{\mathrm{Li}}$ and one Vo case), we can propose that although $2 \delta$ lithium capacity can be accessed in the $\mathrm{Li}_{2} \mathrm{MnO}_{3}$ component, only $\delta$ lithium capacity is kinetically accessible from $\mathrm{Li}_{2} \mathrm{MnO}_{3}$. This, along with the irreversible structure change, might contribute to the irreversible capacity after the $\mathrm{Li}_{2} \mathrm{MnO}_{3}$ component is activated. Therefore, the oxygen vacancy amount needs to be well controlled during the activation process. 


\section{Conclusions}

DFT calculations were combined with AIMD calculations to study the effect that oxygen vacancies have on the formation of lithium vacancies and the diffusion of lithium. The most energetically favorable locations of the $\mathrm{V}_{\mathrm{Li}}$ in systems already containing $\mathrm{V}_{\mathrm{O}}$ are in the neighboring positions of the $\mathrm{V}_{\mathrm{O}}$. A trend of increasing energy of formation of the $\mathrm{V}_{\mathrm{Li}}$ with increasing distance from the $V_{O}$ was also observed, suggesting that the $V_{L i}$ closest to the $V_{O}$ would be the first to be removed during the delithiation process and the last to be replaced during the relithiation process. Additionally, the first two $\mathrm{V}_{\mathrm{Li}}$ generated in the system tended to form "dumbbell" structures around each $\mathrm{V}_{\mathrm{O}}$ with one of the $\mathrm{V}_{\mathrm{Li}}$ in the neighboring lithium layer and one in $\mathrm{V}_{\mathrm{Li}}$ the neighboring transition metal layer.

During the AIMD simulations less Li atoms "hopped", i.e. moved to neighboring positions, with oxygen vacancies present. In particular, it was observed that when one $V_{O}$ and one $V_{L i}$ were placed in their energetically most favorable positions only one $\mathrm{V}_{\mathrm{Li}}$ "hopped" and only for less than 5 ps before hopping back to its original position. However, when a second $\mathrm{V}_{\mathrm{Li}}$ was added in the most energetically favorable position, a significant amount of "hopping" of Li atoms was observed. This combined with the DFT results seems to suggest that while the ratio of $\mathrm{V}_{\mathrm{Li}}$ which are energetically accessible in $\mathrm{Li}_{2} \mathrm{MnO}_{3-\delta}$ with to the amount of $\mathrm{V}_{\mathrm{O}}$ created may be $2: 1$, it appears that only a 1:1 ratio may be kinetically accessible and this might contribute, along with structure change, to the irreversible capacity loss observed after the "activation" of the $\mathrm{Li}_{2} \mathrm{MnO}_{3}$ component. The effect of $\mathrm{V}_{\mathrm{O}}$ on lithium diffusion was then quantitatively observed in the energy barrier for lithium migration increasing with $\mathrm{V}_{\mathrm{O}}$ present and the diffusion coefficient decreasing by 5 orders of magnitude with $\mathrm{V}_{\mathrm{O}}$ present. Both the tendency of the $\mathrm{V}_{\mathrm{Li}}$ to form near the $\mathrm{V}_{\mathrm{O}}$ and the decrease in the lithium movement suggest a significant interaction between the $V_{L i}$ and $V_{O}$. 


\section{Acknowledgments}

We gratefully acknowledge the support from National Science Foundation under Grant No.

DMR-1410850 and 1410946 for the collaborative GOALI research. All the calculations were performed on the HPCC at MSU. 


\section{References}

1. M. M. Thackeray et al., Li2MnO3-stabilized LiMO2 ( $\mathrm{M}=\mathrm{Mn}, \mathrm{Ni}, \mathrm{Co})$ electrodes for lithium-ion batteries. Journal of Materials Chemistry 17, 3112-3125 (2007).

2. M. S. Whittingham, Lithium batteries and cathode materials. Chemical Reviews 104, 4271-4301 (2004).

3. T. Ohzuku, R. J. Brodd, An overview of positive-electrode materials for advanced lithium-ion batteries. Journal of Power Sources 174, 449-456 (2007).

4. B. L. Ellis, K. T. Lee, L. F. Nazar, Positive Electrode Materials for Li-Ion and LiBatteries. Chemistry of Materials 22, 691-714 (2010).

5. B. Xu, D. N. Qian, Z. Y. Wang, Y. S. L. Meng, Recent progress in cathode materials research for advanced lithium ion batteries. Materials Science \& Engineering R-Reports 73, 51-65 (2012).

6. Lithium-Ion Batteries: Advanced Materials and Technologies. S. Lee, Ed., Green Chemistry and Chemical Engineering (CRC Press, Boca Raton, Florida, 2012), pp. 406.

7. S. Hy, F. Felix, J. Rick, W. N. Su, B. J. Hwang, Direct In situ Observation of Li2O Evolution on Li-Rich High-Capacity Cathode Material, Li NixLi(1-2x)/3Mn(2-x)/3 O-2 $(0<=\mathrm{x}<=0.5)$. Journal of the American Chemical Society 136, 999-1007 (2014).

8. A. R. Armstrong, P. G. Bruce, Electrochemistry beyond Mn4+ in LixMn1-yLiyO2. Electrochemical and Solid State Letters 7, A1-A4 (2004).

9. A. R. Armstrong et al., Demonstrating oxygen loss and associated structural reorganization in the lithium battery cathode Li Ni0.2Li0.2Mn0.6 O-2. Journal of the American Chemical Society 128, 8694-8698 (2006).

10. J. R. Croy et al., Examining Hysteresis in Composite $\mathrm{xLi}(2) \mathrm{MnO}(3) \operatorname{center} \operatorname{dot}(1-$ x)LiMO2 Cathode Structures. Journal of Physical Chemistry C 117, 6525-6536 (2013).

11. J. R. Croy, K. G. Gallagher, M. Balasubramanian, B. R. Long, M. M. Thackeray, Quantifying Hysteresis and Voltage Fade in $\mathrm{xLi}(2) \mathrm{MnO}(3)$ center $\operatorname{dot}(1-$ $\mathrm{x}) \mathrm{LiMn} 0.5 \mathrm{Ni} 0.5 \mathrm{O} 2$ Electrodes as a Function of Li2MnO3 Content. Journal of the Electrochemical Society 161, A318-A325 (2014).

12. K. G. Gallagher et al., Correlating hysteresis and voltage fade in lithium- and manganeserich layered transition-metal oxide electrodes. Electrochemistry Communications 33, 9698 (2013).

13. X. Q. Yu et al., Understanding the Rate Capability of High-Energy-Density Li-Rich Layered Li $1.2 \mathrm{Ni} 0.15$ Co $0.1 \mathrm{Mn} 0.55 \mathrm{O} 2$ Cathode Materials. Advanced Energy Materials 4, 11 (2014).

14. A. Boulineau et al., Evolutions of Li1.2Mn0.61 Ni0.18Mg0.01O2 during the Initial Charge/Discharge Cycle Studied by Advanced Electron Microscopy. Chemistry of Materials 24, 3558-3566 (2012).

15. P. Kalyani, S. Chitra, T. Mohan, S. Gopukumar, Lithium metal rechargeable cells using Li2MnO3 as the positive electrode. Journal of Power Sources 80, 103-106 (1999). 
16. G. R. Jain, J. S. Yang, M. Balasubramanian, J. J. Xu, Synthesis, electrochemistry, and structural studies of lithium intercalation of a nanocrystalline Li2MnO3-like compound. Chemistry of Materials 17, 3850-3860 (2005).

17. A. D. Robertson, P. G. Bruce, Mechanism of electrochemical activity in Li2MnO3. Chemistry of Materials 15, 1984-1992 (2003).

18. S. H. Park, Y. Sato, J. K. Kim, Y. S. Lee, Powder property and electrochemical characterization of Li2MnO3 material. Materials Chemistry and Physics 102, 225-230 (2007).

19. P. Strobel, B. Lambertandron, CRYSTALLOGRAPHIC AND MAGNETICSTRUCTURE OF LI2MNO3. Journal of Solid State Chemistry 75, 90-98 (1988).

20. W. P. Tang, H. F. Kanoh, X. J. Yang, K. Ooi, Preparation of plate-form manganese oxide by selective lithium extraction from monoclinic $\mathrm{Li} 2 \mathrm{MnO} 3$ under hydrothermal conditions. Chemistry of Materials 12, 3271-3279 (2000).

21. P. F. Yan et al., Probing the Degradation Mechanism of Li2MnO3 Cathode for Li-Ion Batteries. Chemistry of Materials 27, 975-982 (2015).

22. Y. Paik, C. P. Grey, C. S. Johnson, J. S. Kim, M. M. Thackeray, Lithium and deuterium NMR studies of acid-leached layered lithium manganese oxides. Chemistry of Materials 14, 5109-5115 (2002).

23. M. H. Rossouw, M. M. Thackeray, LITHIUM MANGANESE OXIDES FROM LI2MN03 FOR RECHARGEABLE LITHIUM BATTERY APPLICATIONS. Materials Research Bulletin 26, 463-473 (1991).

24. C. Jacob et al., Electrochemical and Structural Effects of In Situ Li2O Extraction from Li2MnO3 for Li-Ion Batteries. Acs Applied Materials \& Interfaces 7, 2433-2438 (2015).

25. M. Sathiya et al., High Performance Li2Ru1-yMnyO3 $(0.2<=\mathrm{y}<=0.8)$ Cathode Materials for Rechargeable Lithium-Ion Batteries: Their Understanding. Chemistry of Materials 25, 1121-1131 (2013).

26. A. Ito et al., In situ X-ray absorption spectroscopic study of Li-rich layered cathode material Li Ni0.17Li0.2Co0.07Mn0.56 O-2. Journal of Power Sources 196, 6828-6834 (2011).

27. Y. S. Hong, Y. J. Park, K. S. Ryu, S. H. Chang, M. G. Kim, Synthesis and electrochemical properties of nanocrystalline $\mathrm{Li} \operatorname{NixLi}(1-2 \mathrm{x}) / 3 \mathrm{Mn}(2-\mathrm{x}) / 3 \mathrm{O}-2$ prepared by a simple combustion method. Journal of Materials Chemistry 14, 1424-1429 (2004).

28. E. Lee, K. A. Persson, Structural and Chemical Evolution of the Layered Li-Excess LixMnO3 as a Function of Li Content from First-Principles Calculations. Advanced Energy Materials 4, 8 (2014).

29. R. J. Xiao, H. Li, L. Q. Chen, Density Functional Investigation on Li2MnO3. Chemistry of Materials 24, 4242-4251 (2012).

30. Y. Koyama, I. Tanaka, M. Nagao, R. Kanno, First-principles study on lithium removal from Li2MnO3. Journal of Power Sources 189, 798-801 (2009). 
31. Y. Okamoto, Ambivalent Effect of Oxygen Vacancies on Li2MnO3: A First-Principles Study. Journal of the Electrochemical Society 159, A152-A157 (2012).

32. Y. C. Chen et al., Structural, Electrical, and Lithium Ion Dynamics of Li2MnO3 from Density Functional Theory. Chinese Physics Letters 32, 5 (2015).

33. K. Kubota et al., Direct synthesis of oxygen-deficient Li2MnO3-x for high capacity lithium battery electrodes. Journal of Power Sources 216, 249-255 (2012).

34. R. C. Longo et al., Phase stability of Li-Mn-O oxides as cathode materials for Li-ion batteries: insights from ab initio calculations. Physical Chemistry Chemical Physics 16, 11218-11227 (2014).

35. K. J. Carroll et al., Probing the electrode/electrolyte interface in the lithium excess layered oxide Li1.2Ni0.2Mn0.6O2. Physical Chemistry Chemical Physics 15, 1112811138 (2013).

36. B. Xu, C. R. Fell, M. F. Chi, Y. S. Meng, Identifying surface structural changes in layered Li-excess nickel manganese oxides in high voltage lithium ion batteries: A joint experimental and theoretical study. Energy \& Environmental Science 4, 2223-2233 (2011).

37. S. H. Park, S. H. Kang, C. S. Johnson, K. Amine, M. M. Thackeray, Lithium-manganesenickel-oxide electrodes with integrated layered-spinel structures for lithium batteries. Electrochemistry Communications 9, 262-268 (2007).

38. J. M. Paulsen, C. L. Thomas, J. R. Dahn, Layered Li-Mn-oxide with the O2 structure: A cathode material for Li-ion cells which does not convert to spinel. Journal of the Electrochemical Society 146, 3560-3565 (1999).

39. J. Reed, G. Ceder, A. Van der Ven, Layered-to-spinel phase transition in LixMnO2. Electrochemical and Solid State Letters 4, A78-A81 (2001).

40. D. L. Ye et al., Ni-induced stepwise capacity increase in Ni-poor Li-rich cathode materials for high performance lithium ion batteries. Nano Research 8, 808-820 (2015).

41. D. N. Qian, B. Xu, M. F. Chi, Y. S. Meng, Uncovering the roles of oxygen vacancies in cation migration in lithium excess layered oxides. Physical Chemistry Chemical Physics 16, 14665-14668 (2014).

42. H. J. Yu et al., Electrochemical kinetics of the 0.5 Li(2) MnO(3)center dot $0.5 \mathrm{LiMn}(0.42) \mathrm{Ni}(0.42) \mathrm{Co}(0.16) \mathrm{O}(2)$ 'composite' layered cathode material for lithium-ion batteries. Rsc Advances 2, 8797-8807 (2012).

43. S. Shi et al., Direct Calculation of Li-Ion Transport in the Solid Electrolyte Interphase. Journal of the American Chemical Society 134, 15476-15487 (2012).

44. S. Shi, Y. Qi, H. Li, L. G. Hector, Jr., Defect Thermodynamics and Diffusion Mechanisms in Li2CO3 and Implications for the Solid Electrolyte Interphase in Li-Ion Batteries. Journal of Physical Chemistry C 117, 8579-8593 (2013).

45. C. R. Fell et al., Correlation Between Oxygen Vacancy, Microstrain, and Cation Distribution in Lithium-Excess Layered Oxides During the First Electrochemical Cycle. Chemistry of Materials 25, 1621-1629 (2013). 
46. G. Kresse, J. Hafner, ABINITIO MOLECULAR-DYNAMICS FOR LIQUID-METALS. Physical Review B 47, 558-561 (1993).

47. G. Kresse, J. Hafner, AB-INITIO MOLECULAR-DYNAMICS SIMULATION OF THE LIQUID-METAL AMORPHOUS-SEMICONDUCTOR TRANSITION IN GERMANIUM. Physical Review B 49, 14251-14269 (1994).

48. G. Kresse, J. Furthmuller, Efficient iterative schemes for ab initio total-energy calculations using a plane-wave basis set. Physical Review B 54, 11169-11186 (1996).

49. G. Kresse, J. Furthmuller, Efficiency of ab-initio total energy calculations for metals and semiconductors using a plane-wave basis set. Computational Materials Science 6, 15-50 (1996).

50. P. E. Blochl, PROJECTOR AUGMENTED-WAVE METHOD. Physical Review B 50, 17953-17979 (1994).

51. G. Kresse, D. Joubert, From ultrasoft pseudopotentials to the projector augmented-wave method. Physical Review B 59, 1758-1775 (1999).

52. J. P. Perdew, K. Burke, M. Ernzerhof, Generalized gradient approximation made simple. Physical Review Letters 77, 3865-3868 (1996).

53. J. P. Perdew, K. Burke, M. Ernzerhof, Generalized gradient approximation made simple (vol 77, pg 3865, 1996). Physical Review Letters 78, 1396-1396 (1997).

54. S. Nose, A UNIFIED FORMULATION OF THE CONSTANT TEMPERATURE MOLECULAR-DYNAMICS METHODS. Journal of Chemical Physics 81, 511-519 (1984).

55. G. Henkelman, B. P. Uberuaga, H. Jonsson, A climbing image nudged elastic band method for finding saddle points and minimum energy paths. Journal of Chemical Physics 113, $9901-9904$ (2000).

56. G. Henkelman, H. Jonsson, Improved tangent estimate in the nudged elastic band method for finding minimum energy paths and saddle points. Journal of Chemical Physics 113, 9978-9985 (2000).

57. S. Q. Shi et al., Direct Calculation of Li-Ion Transport in the Solid Electrolyte Interphase. Journal of the American Chemical Society 134, 15476-15487 (2012).

58. K. Kang, D. Morgan, G. Ceder, First principles study of Li diffusion in I-Li2NiO2 structure. Physical Review B 79, 4 (2009).

59. A. Van der Ven, G. Ceder, First principles calculation of the interdiffusion coefficient in binary alloys. Physical Review Letters 94, 4 (2005).

60. A. Van der Ven, G. Ceder, Lithium diffusion mechanisms in layered intercalation compounds. Journal of Power Sources 97-8, 529-531 (2001).

61. W. Frank, U. Breier, C. Elsasser, M. Fahnle, First-principles calculations of absolute concentrations and self-diffusion constants of vacancies in lithium. Physical Review Letters 77, 518-521 (1996).

62. C. P. Flynn, ATOMIC MIGRATION IN MONATOMIC CRYSTALS. Physical Review 171, 682-\& (1968). 
63. J. Sugiyama et al., Antiferromagnetic spin structure and lithium ion diffusion in Li2MnO3 probed by mu+SR. Physical Review B 87, 11 (2013).

64. J. Sugiyama et al., Lithium diffusive behavior in $\mathrm{Li} 2 \mathrm{MnO} 3$ detected by muon-spin relaxation. Solid State Ionics 262, 901-903 (2014).

65. P. G. Shewmon, Diffusion in solids. (Minerals, Metals \& Materials Society, Warrendale, ed. 2nd ed., 1989). 
Table 1: Calculated $\mathrm{Li}_{2} \mathrm{MnO}_{3}$ lattice parameters compared to previously calculated parameters, by Koyama, et al.(30) and Okamoto,(31) and experimentally determined parameters by Strobel et al.(19)

\begin{tabular}{l|lll|l}
\hline \multirow{2}{*}{ Lattice } & \multicolumn{3}{|c|}{ Calculated $(\AA)$} & Experimental $(\AA)$ \\
\cline { 2 - 4 } & Current & Koyama et al. & Okamoto & Strobel, et al. \\
\hline a & calculation & & \\
b & 5.01 & 5.02 & 4.98 & 4.94 \\
c & 8.66 & 8.68 & 8.63 & 8.53 \\
\hline
\end{tabular}




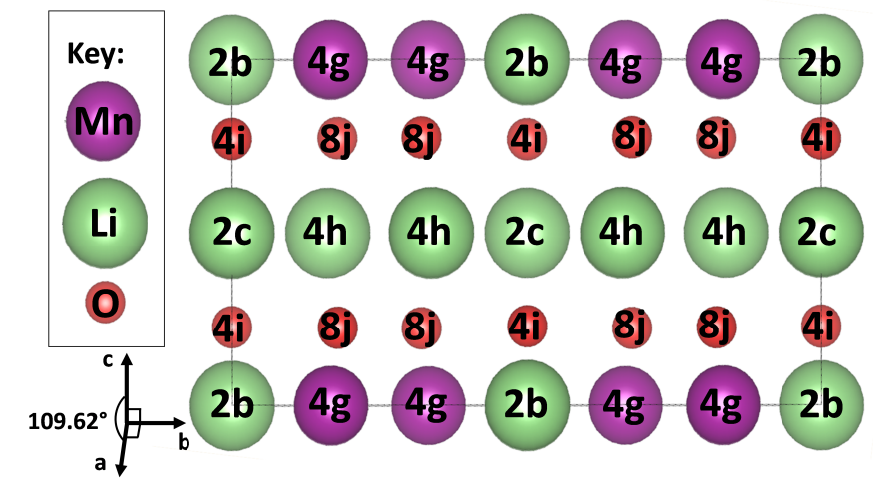

Figure 1: perfect $\mathrm{Li}_{2} \mathrm{MnO}_{3}$ unit cell projected along [100] direction, serving as the staring structure for vacancy generations. The Wyckoff positions are depicted. (color online, black and white print) 
(a)

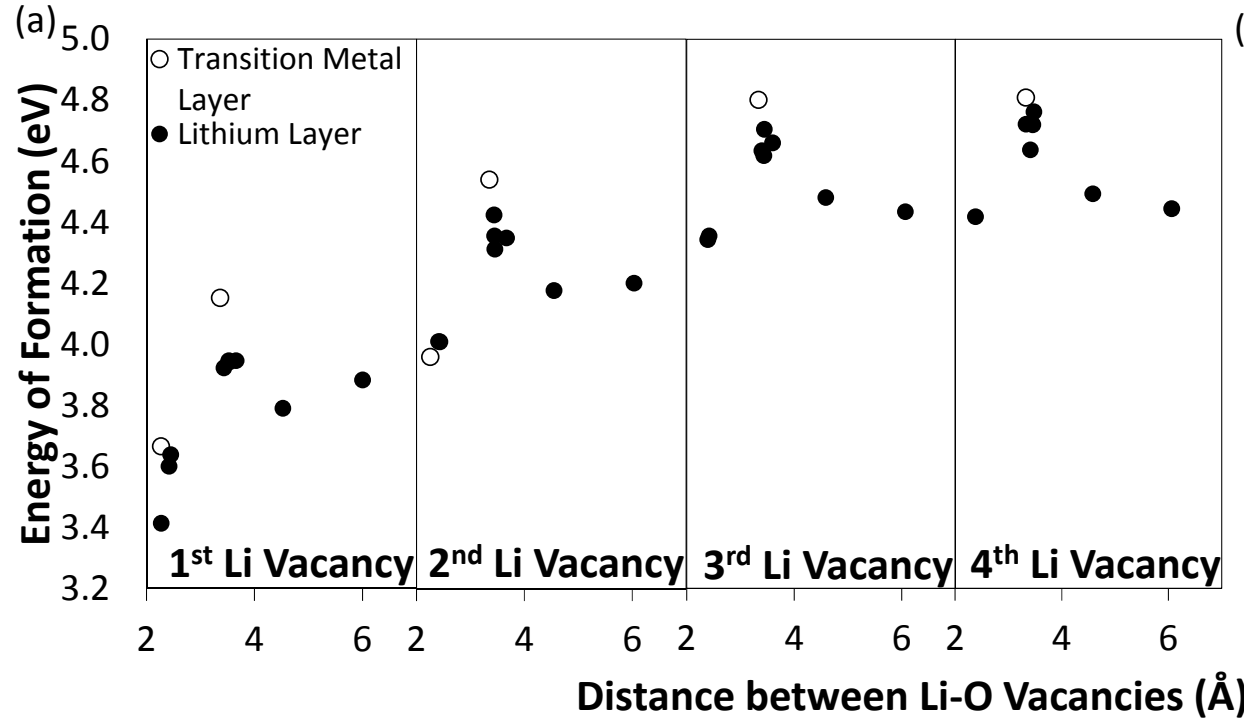

(b) 4.5

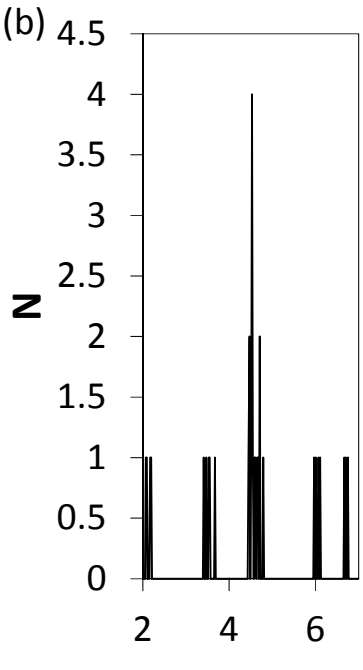

(c)

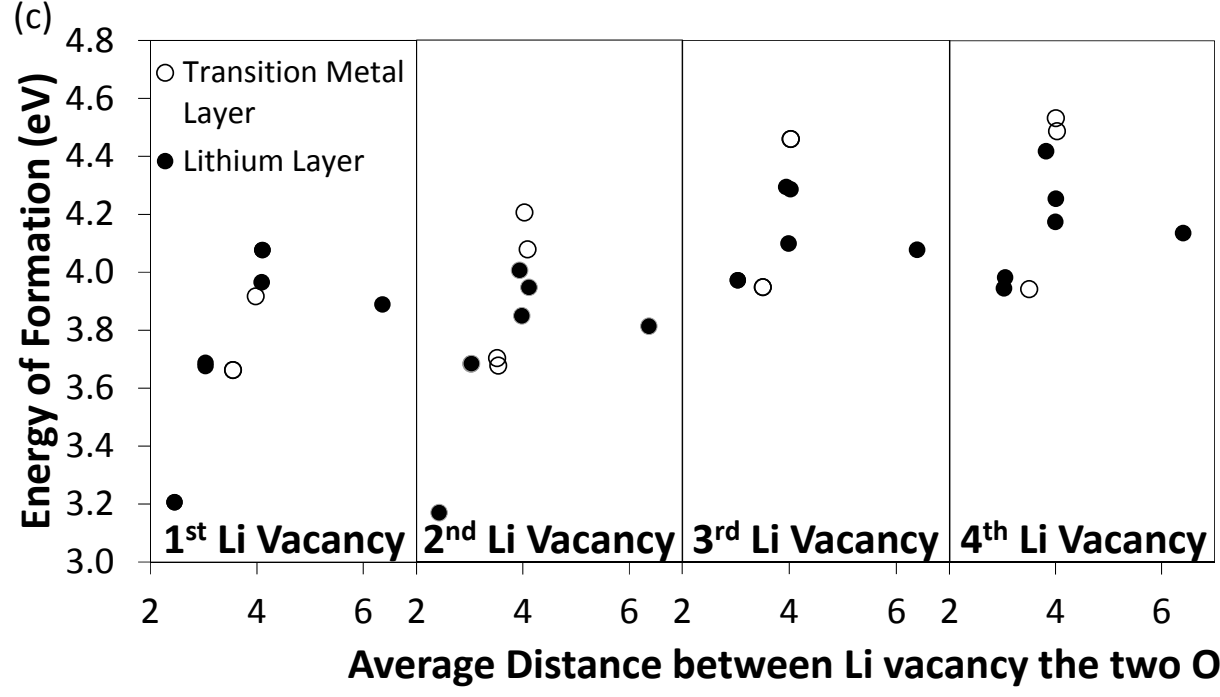

(d)

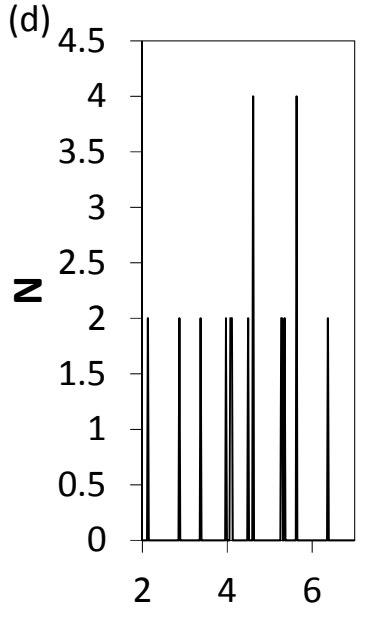

Figure 2: Comparison of DFT calculated energy of formation for creating $\mathrm{V}_{\mathrm{Li}}$, one at a time from previously optimized structures containing (a) a single $V_{O}$ and (c) two Vo. The solid and open symbols indicate the $\mathrm{Li}$ was removed from the Li layer or the transition metal layer, respectively. (b) \& (d) show the number of Li atoms vs. the distances for (a) and (c), respectively. In (a) and (b), the distance is between the $\mathrm{V}_{\mathrm{Li}}$ and the single $\mathrm{V}_{\mathrm{O}}$ position; and in (b) and (d), the distance is between the $\mathrm{V}_{\mathrm{Li}}$ and the center of the two $\mathrm{V}_{\mathrm{O}}$. 
(a)

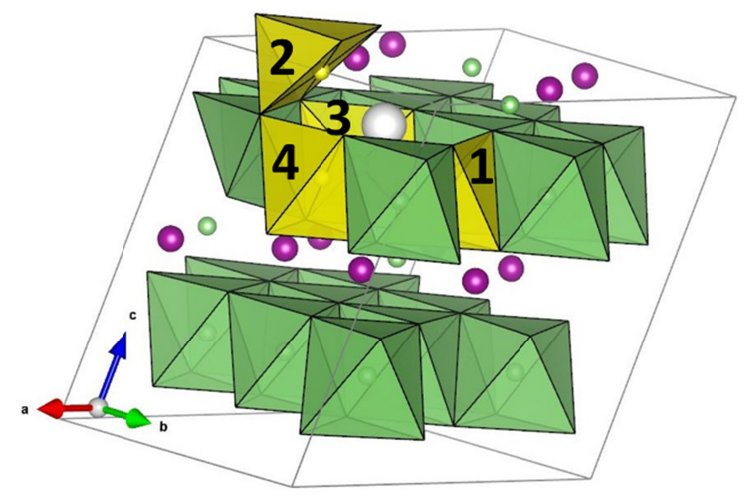

(b)

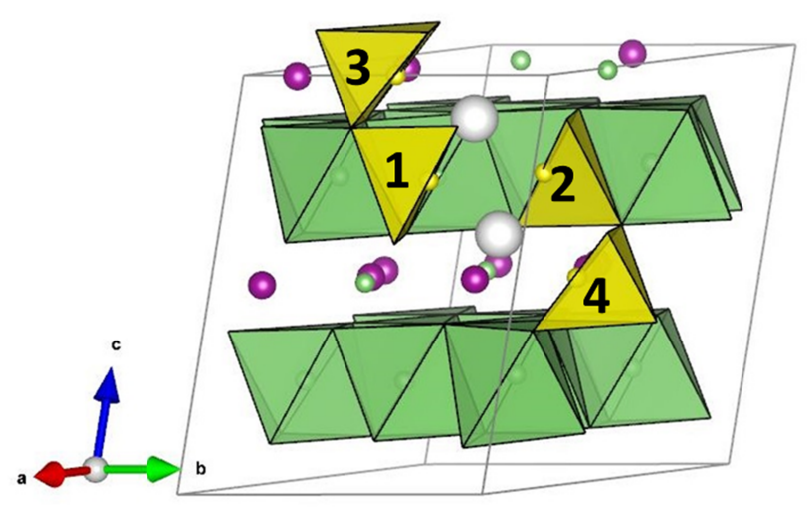

Figure 3: Relaxed atomic positions for systems with $4 \mathrm{~V}_{\mathrm{Li}}$ and either (a) $\mathrm{V}_{\mathrm{O}}$ or (b) two $\mathrm{V}_{\mathrm{O}}$. The yellow Li centered octahedral (or half octahedral due to oxygen vacancy) indicates where the removed $\mathrm{Li}$ atoms were located in the perfect crystal structure and the numbers indicate the sequences they were removed. (color online, black and white print) 
(a)

\begin{tabular}{|lll|}
\hline$o$ & $\ominus$ \\
$v_{0}$ & $\bigcirc$ \\
Li & 0 \\
$v_{i t}$ & 0 \\
Mn & 0 \\
\hline
\end{tabular}

(b)

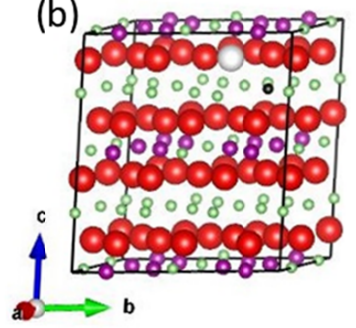

(c)

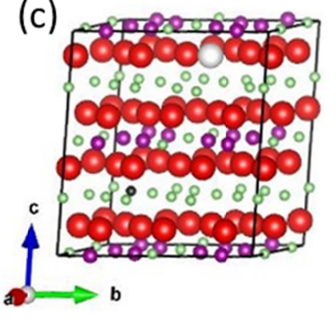

(d)

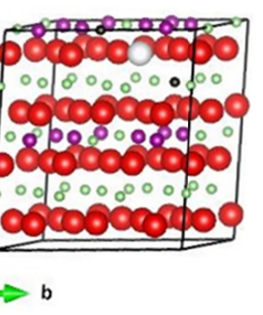

Figure 4: Starting systems for ab-initio molecular dynamics calculations with (a) one $\mathrm{V}_{\mathrm{Li}}$, (b) one $\mathrm{V}_{\mathrm{O}}$ and $\mathrm{V}_{\mathrm{Li}}$, (c) one $\mathrm{V}_{\mathrm{O}}$ and $\mathrm{V}_{\mathrm{Li}}$ farther apart, and (d) one $\mathrm{V}_{\mathrm{O}}$ and two $\mathrm{V}_{\mathrm{Li}}$. (color online, black and white print) 
(a)
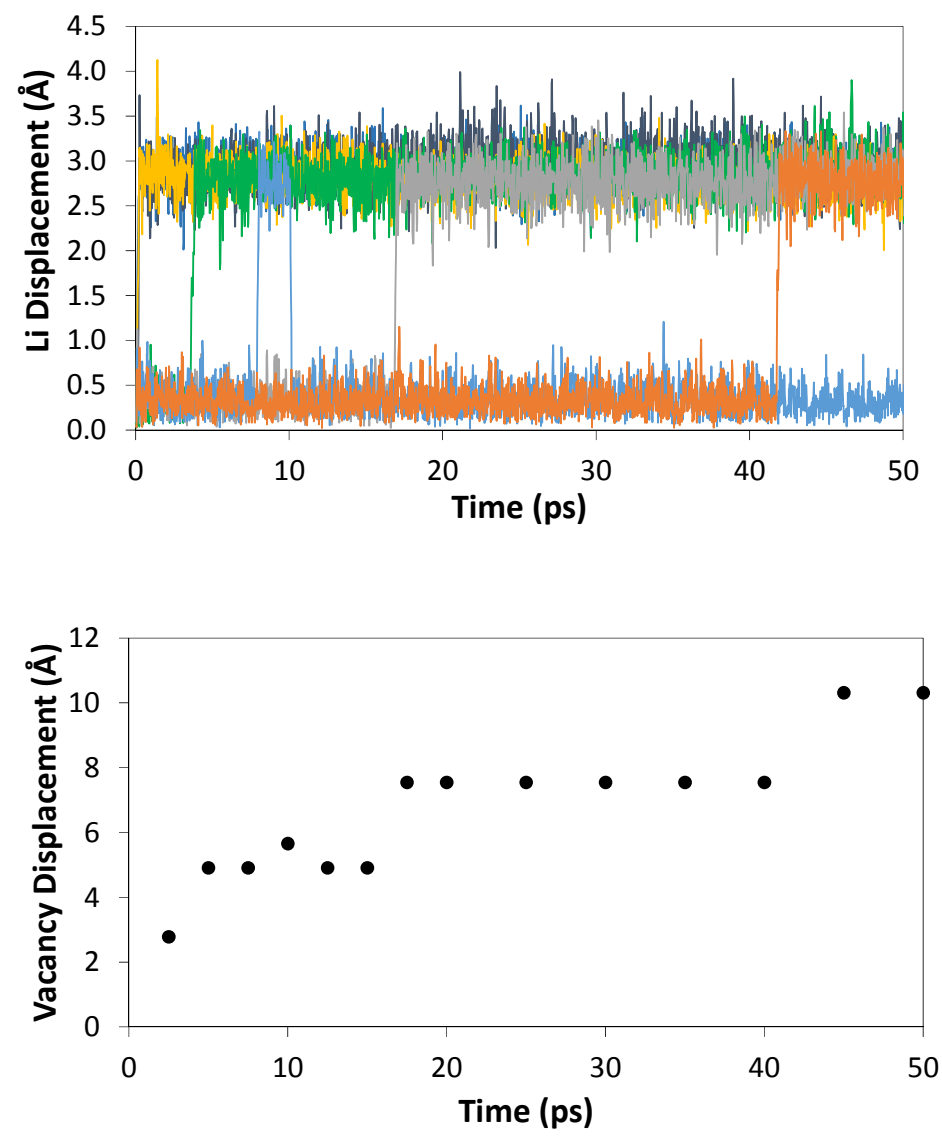

(b)

Figure 5: Displacement of (a) lithium atoms and (b) the $\mathrm{V}_{\mathrm{Li}}$ from their initial positions for a starting system with only one $\mathrm{V}_{\mathrm{Li}}$ and no $\mathrm{V}_{\mathrm{O}}$ (in Figure 4a) during a 50ps AIMD simulation. (color online, black and white print) 
Submit to Solid State Ionics

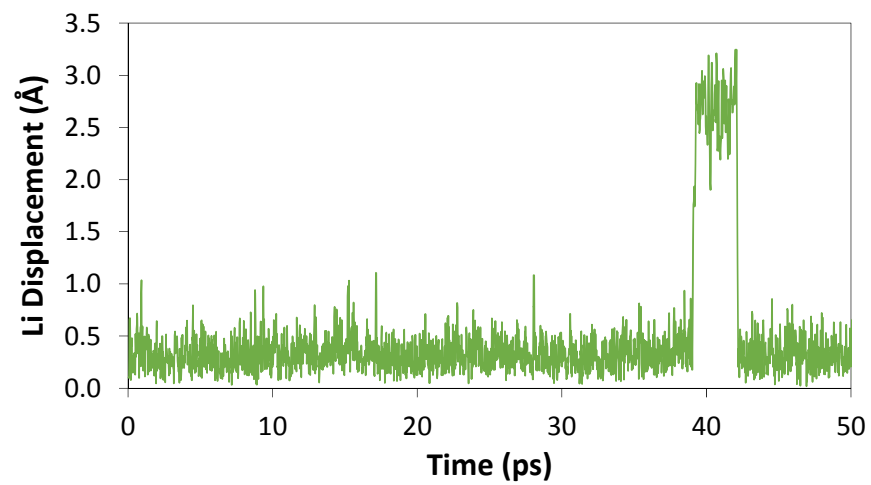

(a)

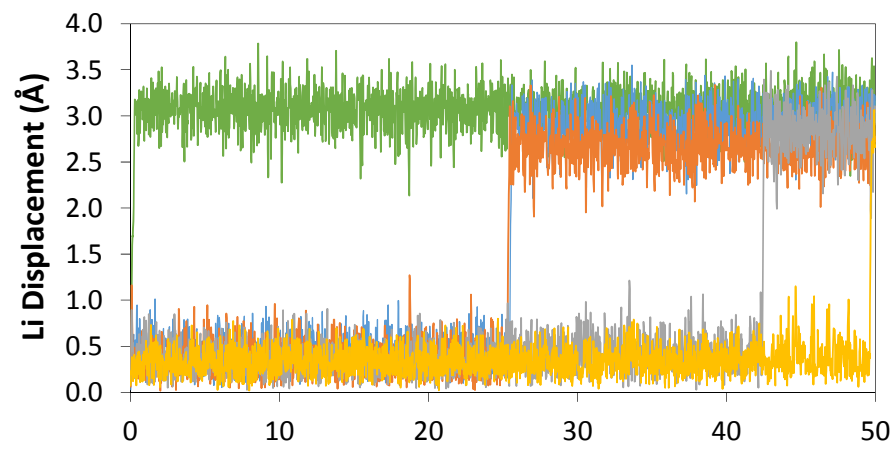

(b)

Time (ps)

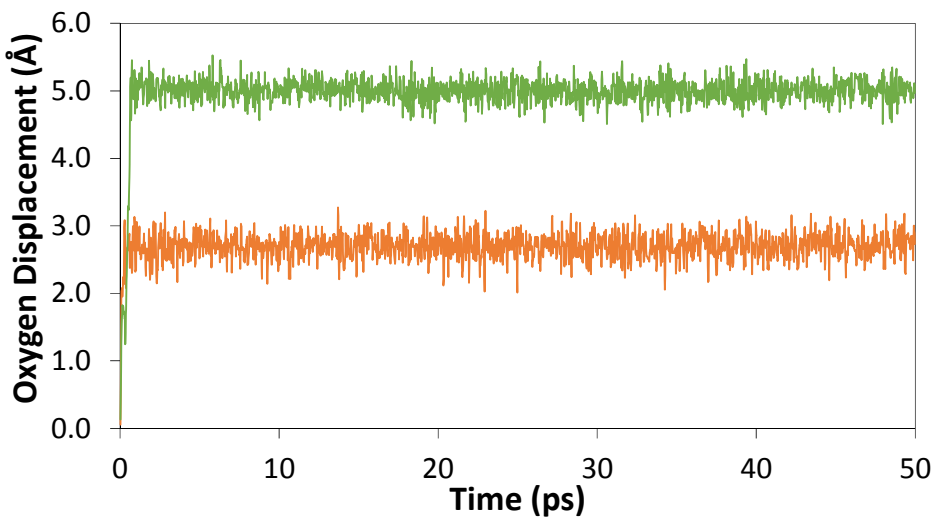

(c)

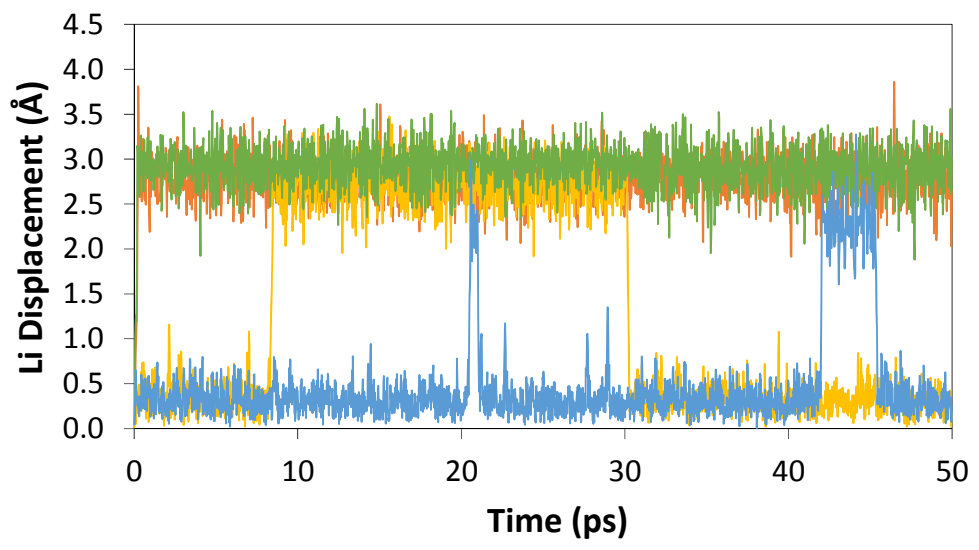


Figure 6: Displacement of lithium and oxygen atoms from their initial positions in the initial structures shown in Figure 4 for 50 ps AIMD simulations. (a): For the starting system with one $\mathrm{V}_{\mathrm{Li}}$ and one $\mathrm{V}_{\mathrm{O}}$, in neighbor positions (shown in Figure $4 \mathrm{~b}$ ), the hopping of only one lithium atom was observed. (b) and (c): For a starting system with one $\mathrm{V}_{\mathrm{Li}}$ and one $\mathrm{V}_{\mathrm{O}}$ placed farther apart (shown in Figure 4c), displacements of both (b) lithium atoms and (c) oxygen atoms were observed. (d): For a starting system with two $V_{L i}$ and one $V_{O}$ (shown in Figure $4 d$ ), there was observed displacement of one of the two lithium atoms. (color online, black and white print) 


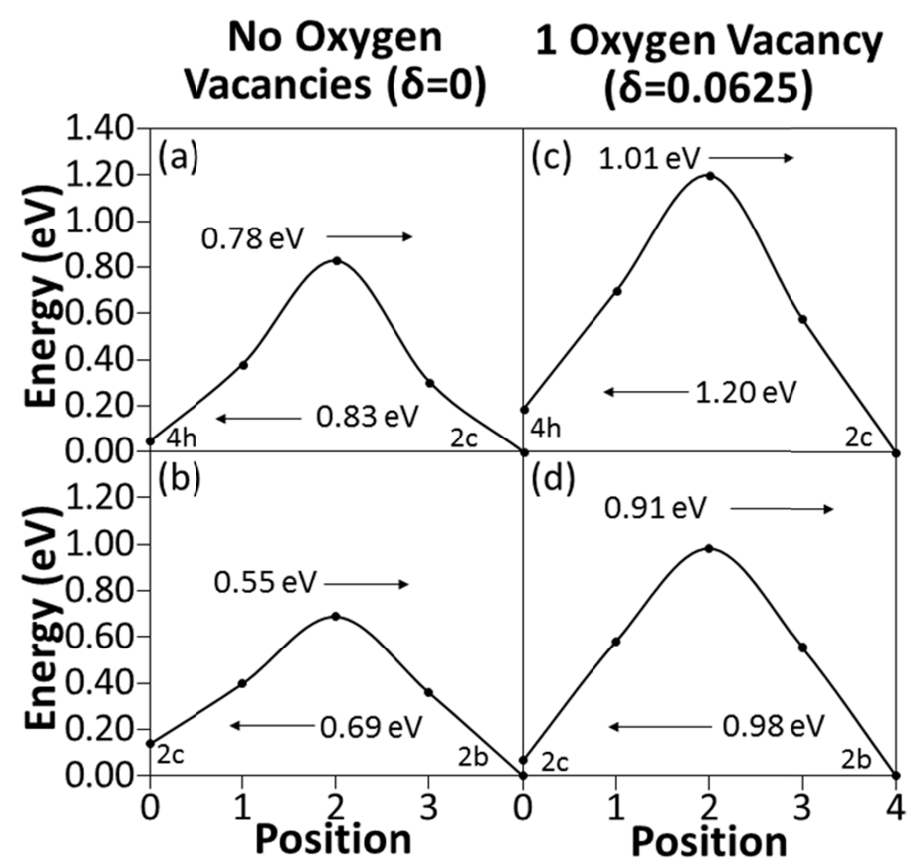

Figure 7: Calculated energy barrier with no oxygen vacancies for (a) cross-layer hopping between the $4 h$ and $2 c$ positions, and (b) lithium-layer hopping between the $2 c$ and $2 b$ positions. The energy barrier was increased by the introduction of one oxygen vacancy in the system for both the hopping between (c) the $4 h$ and $2 c$ positions and (d) the $2 c$ and $2 b$ positions. To show the barrier, the reference energy was set zero for the equilibrium structure with the lowest energy for the four cases separately. 


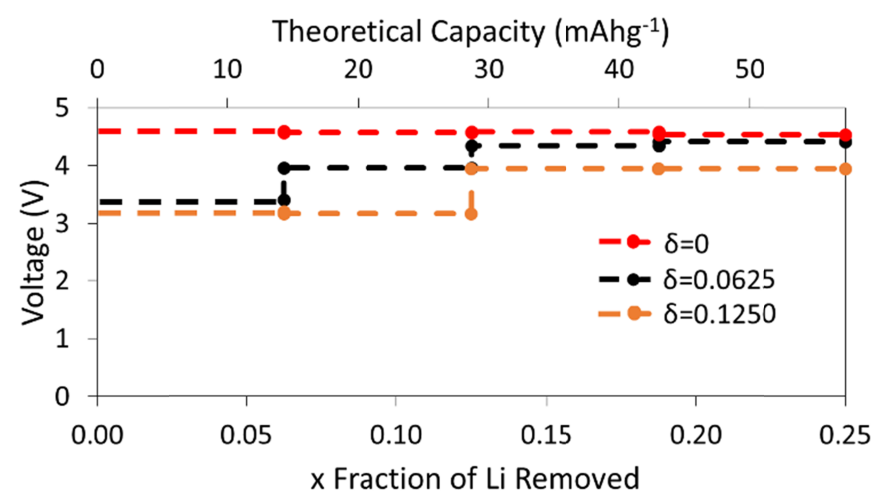

Figure 8: Calculated open circuit voltage curve for $0 \leq x \leq 0.25$ and varying $\delta$ in $\mathrm{Li}_{2-x} \mathrm{MnO}_{3-\delta}$. (color online, black and white print) 